\title{
Propuesta de densificación energética de residuos y recursos biomásicos mediante peletización
}

\section{Proposal for energy densification of waste and biomass resources through pelletization}

\author{
Gregory Marin ${ }^{l}$, Josimar Pérez', Orlando Aguilar ${ }^{*}$ \\ ${ }^{1}$ Estudiantes de Licenciatura en Ingeniería Mecánica, Facultad de Ingeniería Mecánica, Universidad Tecnológica de Panamá, \\ ${ }^{2}$ Profesor, Facultad de Ingeniería Mecánica, Universidad Tecnológica de Panamá
}

\begin{abstract}
Resumen En la actualidad el mundo está en la búsqueda de nuevas formas de aprovechar las energías limpias. Una oportunidad en este sentido lo son los desechos orgánicos producidos por las actividades humanas o de forma natural en el ambiente. Otra oportunidad prometedora son los cultivos energéticos como recursos biomásicos. Este trabajo presenta una propuesta de investigación para la utilización de residuos orgánicos sólidos, tales como papel y cartón, restos de poda de áreas verdes, restos de madera y material biomásico proveniente de la Paja Canalera (un tipo de hierba alta relacionado con la caña de azúcar), para producir un combustible sólido mediante la peletización de dichos materiales. Se evaluarán las propiedades físico-químicas y el poder calorífico por unidad de volumen, del material obtenido.
\end{abstract}

Palabras clave Biomasa, biocombustible sólido, materia orgánica, densificación, pélets, Paja Canalera.

Abstract Currently, the world is searching for new ways to take advantage of clean energy. An opportunity in this sense, are the organic wastes produced by human activities or naturally in the environment. Another promising opportunity is energy crops as biomass resources. This work presents a research proposal for the use of solid organic wastes, such as paper and cardboard, pruning remains, wood remnants and biomass material from Paja Canalera (a tall grass type related to sugar cane), to produce solid fuel by pelletizing said materials. The physical-chemical properties and calorific value per unit of volume of the material obtained will be evaluated.

Keywords Biomass, solid biofuel, organic material, densification, pellets, Paja Canalera.

* Corresponding author: orlando.aguilar@utp.ac.pa

\section{Introducción}

La Universidad Tecnológica de Panamá (UTP) presenta en sus instalaciones una gran cantidad de residuos sólidos que pueden ser usados como recurso biomásico. Estos materiales pueden ser sometidos a un proceso de compactación llamado peletización. Mediante este proceso, un gran volumen de material puede compactarse en un volumen mucho más pequeño, con lo cual, la densidad del material aumenta drásticamente, aumentando también así, su densidad energética. Entre los residuos se puede mencionar en el caso del campus Víctor Levi Sasso, restos de jardinería, que se componen de gran cantidad de hojas y restos provenientes de los árboles circundantes en el Campus. Además, se conoce que el campus de la UTP genera un promedio de $755 \mathrm{~kg}$ de residuos sólidos diariamente, los cuales pueden ser adecuados mediante distintos procesos para su aprovechamiento energético [1].

La peletización de biocombustibles sólidos para uso térmico constituye una opción real para la revalorización de los residuos. En los últimos años, la producción y comercialización de productos biomásicos densificados ha experimentado un significativo aumento [2].

Los pélets son elementos densificados de forma cilíndrica, con diámetros entre 4 y $12 \mathrm{~mm}$ y longitudes de 10 a $30 \mathrm{~mm}$, utilizados como combustible. Si esos pélets se producen a partir de residuos orgánicos, presentan ventajas frente a otros tipos de combustible, ya que se trata de una fuente de energía renovable con balance neutro de $\mathrm{CO}_{2}$, por lo que su uso contribuye a la reducción significativa de la emisión de gases de efecto invernadero a la atmósfera. Por todo ello, la biomasa densificada representa un combustible de cierta relevancia en todo el mundo [3].

\subsection{Generalidades y terminología}

El concepto de biomasa comprende todo tipo de materia orgánica, tanto de origen vegetal como animal, y que está formada gracias a la fotosíntesis directamente (como los vegetales) o indirectamente (por la digestión de los vegetales). Por su propia definición, la biomasa abarca un amplio conjunto de productos orgánicos que se caracterizan por su heterogeneidad tanto en lo referente a su origen como a su 
naturaleza intrínseca, si bien puede afirmarse que cualquiera definición de biomasa debe englobar obligatoriamente dos términos: orgánico y renovable [4].

La utilización de biomasa para calefacción y la generación energética se está volviendo cada vez más importante, debido al deseo de reducir las emisiones de dióxido de carbono a la átmosfera. Debido a la gran disponibilidad de biomasa de origen vegetal, esta puede ser utilizada como fuente sostenible cuando se emplea como combustible. Sin embargo, en ciertas ocasiones, se presenta una distancia considerable entre la fuente de suministro y el lugar de utilización, esto crea un costo adicional en lo referente al manejo y transporte del material.

Materiales de origen vegetal como lo son la madera, tienen la tendencia de poseer una consistencia porosa y densidades bajas. Para incrementar dicha densidad, el material puede ser comprimido a forma de pélet, utilizando un proceso mecánico en el que la presión es aplicada al material con el propósito de aplastar su estructura celular y de ese modo incrementar su densidad.

Los mayores beneficios de la peletización son: mayores densidades, menores costos de transporte y almacenaje, composición y tamaño estandarizado del material (lo que permite automatización en los procesos de alimentación a las calderas).

Los materiales más comunes utilizados en la peletización son residuos de madera (como lo son el aserrín), residuos agrarios (como lo es la paja), productos de desecho de la industria alimenticia y cultivos de combustible (como lo son los usados para la producción de bioetanol y similares).

\subsection{Parámetros fundamentales en los pélets}

Un buen combustible sólido y en especial, los que son producidos por recursos biomásicos, debe presentar características o parámetros que garanticen que sean óptimos para su producción. A continuación, se menciona la definición y los parámetros más importantes de los pélets y se destaca que no son los únicos parámetros de medición, pero analizar estos mismos parámetros antes de completar el proceso de peletización es de suma importancia para comparar la factibilidad del proceso, ya que en la actualidad es costoso y conlleva a energía adicional para su producción:

$\checkmark$ Humedad: Presente en un residuo suele estar determinada por el contenido de agua de la biomasa y por el proceso de separación o extracción del residuo biomásico del producto primario. El contenido de humedad influye en el comportamiento de la combustión, así como en la temperatura adiabática de la llama y en el volumen de gases por unidad de energía producida. Por lo tanto, se trata de un parámetro que afecta directamente a la eficacia de la combustión [5].

$\checkmark$ Densidad aparente: La densidad real de un producto biomásico se define como el cociente entre la masa y el volumen original, mientras que la densidad aparente es la correspondiente al volumen ocupado en la práctica, incluyendo tanto huecos como espacios vacíos en el almacenamiento. La mayoría de los residuos biomásicos presentan densidades aparentes bajas, debido a su estructura porosa, con valores que van desde 40 a 150 $\mathrm{kg} / \mathrm{m}^{3}$ para las biomasas herbáceas, que en este estudio son los más importantes. Una elevada densidad aparente supone una considerable ventaja respecto a otros productos, ya que permite almacenar una mayor cantidad de energía en un mismo volumen.

$\checkmark \quad$ Distribución granulométrica: Es una característica muy importante de los residuos biomásicos y afecta al dimensionamiento de los sistemas de almacenamiento y transporte de los mismos, así como el proceso de densificación y consecuentemente al diseño del sistema de combustión. El tamaño y la forma de las partículas influyen directamente en las propiedades físicas, químicas y mecánicas, tanto de los residuos originales como de los pélets fabricados [6].

$\checkmark$ Análisis elemental: El conocimiento de la composición elemental de un residuo biomásico resulta de gran importancia para estimar las propiedades térmicas como potencial combustible. Se tiene un estudio que establece relaciones directas entre el poder calorífico y porcentajes de carbono, hidrógeno y oxígeno [7]. Además, el análisis elemental permite detectar posibles elementos generadores de emisiones nocivas como el nitrógeno y el azufre.

$\checkmark$ Poder calorífico: Es una medida de la energía química intrínseca en un biocombustible, energía que se libera durante la combustión. Es una propiedad muy importante de los combustibles sólidos, ya que se emplea para el diseño y explotación de calderas.

\section{Materiales y métodos}

\subsection{Materiales}

Esta investigación se está llevando a cabo en las instalaciones de la Universidad Tecnológica de Panamá, Extensión de Tocumen, Panamá, República de Panamá. Los materiales que serán procesados consisten principalmente de residuos generados en las instalaciones de la UTP, tales como papel, cartón, madera, hojas de árbol secas, restos de poda, así como biomasa de Paja Canalera, cascarilla de arroz, caña de azúcar y aserrín de madera, entre otros. Los residuos serán triturados mediante una Picadora/Trituradora de forraje, marca Trapp, modelo TRF-300, con un motor monofásico de 2CV @ $60 \mathrm{~Hz}$ de $110 \mathrm{~V}$. Posteriormente serán compactados en pélets mediante un equipo peletizador con una capacidad global de $75-100 \mathrm{~kg} / \mathrm{h}$ y con un dado o matriz con orificios de $5 \mathrm{~mm}$ de diámetro, impulsada por un motor eléctrico de $2.2 \mathrm{~kW}$ monofásico de $110 \mathrm{~V}$. El dado o matriz puede modificarse para variar el diámetro de los pellets.

El poder calorífico de los diferentes materiales se medirá mediante una bomba calorimétrica IKA C2000. La humedad asociada al material al momento de peletizar se medirá haciendo uso de un horno secador y una balanza electrónica. 


\subsection{Metodología}

La investigación tiene como eje principal cuantificar y analizar de forma física y química la calidad del combustible sólido obtenido a partir de la biomasa a través de la técnica de peletización. Para tal finalidad se seguirá la siguiente metodología:

$\checkmark$ Se realizará análisis próximo de los recursos biomásicos a utilizar, en el cual se determina el contenido de humedad, cenizas, materia volátil y carbón fijo expresados en porcentaje en peso del material, siguiendo los procedimientos normados por ASTM.

$\checkmark$ Se realizará un proceso de pretratamiento a los recursos biomásicos previo al proceso de trituración y peletización.

$\checkmark$ Se evaluará diversas humedades y niveles de trituración para tener el material con tamaños uniformes para el posterior proceso de peletización.

$\checkmark \quad$ El material será secado al aire y se llevará un control diario de su humedad hasta alcanzar los niveles de diseño para peletización.

$\checkmark$ Una vez alcanzados la humedad y tamaño de grano adecuados, se realizará el proceso de peletización, siguiendo estándares de producción de pélets.

$\checkmark \quad$ Los pélets serán almacenados en un contenedor seco para su posterior análisis energético mediante combustión y evaluación del poder calorífico.

$\checkmark$ Colección y análisis de datos.

$\checkmark$ Presentación de resultados.

\section{Muestreo y experimentación}

En este estudio se utilizarán tres recursos biomásicos los cuales son las hojas de los bosques y parques que rodean la Universidad Tecnológica de Panamá (UTP), la especie invasora Paja Canalera y los residuos de aserrín provenientes de los laboratorios de la UTP.

\subsection{Muestreo y proceso de experimentación}

Se agruparán los tres tipos de biomasas que se tendrán en el estudio y se analizarán cualitativamente las mismas.

Primero se debe reducir el tamaño de la partícula del material como procedimiento previo al peletizado. Para dicho propósito se empleará una Picadora/Trituradora de material forrajero marca Trapp modelo TRF-300. Esta cuenta con un conjunto de 10 martillos y 2 cuchillas para motor monofásico de 2CV @ 60Hz de 110V. Se tiene la opción de utilizar alguno de los cuatro tamices incluidos con la misma: $0.8,3,5$ y $12 \mathrm{~mm}$ y así controlar el tamaño de los granos (granulometría) y hacer más uniforme las partículas. Dependiendo del tipo de material con el que se vaya a trabajar sea hojas o granos, se tiene diferentes capacidades de triturado. Para ración verde de 700 a $1400 \mathrm{~kg} / \mathrm{h}$, trozos gruesos de 200 a $500 \mathrm{~kg} / \mathrm{h}$, para grano quebrado de 300 a $820 \mathrm{~kg} / \mathrm{h}$, para granos finos de 150 a 300 $\mathrm{kg} / \mathrm{h}$ y para hojuelas finas de 50 a $80 \mathrm{~kg} / \mathrm{h}$. Después se debe medir la humedad que se tiene en las muestras. Se realizarán grupos de muestras variando la humedad utilizando un secado previo para variar los niveles y la otra variable será el tamaño de los granos y su distribución para luego proceder a peletizar las muestras.

Para tal finalidad se utilizará una peletizadora que tiene una capacidad global de $75-100 \mathrm{~kg} / \mathrm{h}$ y cuenta con un dado con orificios de $4 \mathrm{~mm}$ de diámetro. Esta máquina es impulsada por un motor eléctrico de $2.2 \mathrm{~kW}$ monofásico de $110 \mathrm{~V}$. El dado o matriz puede modificarse para variar los diámetros de los pélets, pero en este estudio se mantendrán fijas.

\subsection{Ecuaciones}

En el estudio de los pélets como se ha indicado anteriormente, influyen muchos parámetros, para este caso se utilizarán equipos para medir y hacer un análisis estadístico entre una variable y dos parámetros constates, específicamente la variable será la humedad, que tiene una relación estrella con la masa y la durabilidad, por lo que estos parámetros se medirán de forma experimental y teórico con las siguientes ecuaciones:

La durabilidad de los pellets se expresa en PDI (Índice de Durabilidad de Pellets por sus siglas en inglés). Esto representa la cantidad de material que se desprende del pellet debido a presiones neumáticas o mecánicas a las cuales son sometidos. El PDI se calcula como un porcentaje de peso antes y después de la medida.

$$
P D I=\frac{m_{2}}{m_{1}} * 100
$$

$m_{1}$ se toma como la masa antes de la prueba,

$m_{2}$ como la masa después.

La dureza representa la fuerza necesaria para aplastar el pellet. Los pellets con mayor dureza tienen mejor calidad y mayor masa por unidad de volumen.

El nivel de humedad en los pélets se expresa como índice WI (Wettability Index). Este se mide pesando un pélet y luego introduciéndolo en agua durante $30 \mathrm{~s}$ para ser pesado nuevamente. El índice se calcula como el porcentaje de la diferencia de peso antes y después de la prueba.

$$
W I=\frac{m_{2}-m_{1}}{m_{1}} * 100
$$

\section{Resultados y discusión}

De este estudio se espera cuantificar los residuos sólidos generados en la Universidad Tecnológica de Panamá con capacidad de ser utilizados como combustible sólido, mediante su densificación energética a través de peletización. Se espera además, la caracterización de los pélets mediante análisis de las propiedades físicas, químicas y energéticas, especialmente 
Marin (et al): Propuesta de densificación energética de residuos y recursos biomásicos mediante peletización

aquellas cuya influencia será mayor en el proceso de combustión como la humedad y su influencia en los demás parámetros importantes para obtener un pellet adecuado, en base a normativa europea.

Al densificar esos residuos sólidos, se mejorarán sus propiedades fisicoquímicas, resultando en un combustible sólido útil y obtenido a partir de residuos, que, de otra forma, irían a parar al vertedero municipal de la Ciudad de Panamá. Mediante este estudio, se generará información útil sobre el comportamiento térmico de los productos densificados, de donde se podrá observar las ventajas económicas y energéticas en comparación con el material crudo (no densificado).

Finalmente, este estudio brindará información que posibilite la exploración de nuevos recursos como combustible sólido alternativo para uso en calderas y hornos, así como en otras aplicaciones industriales.

\section{Conclusiones preliminares}

La peletización figura como una viabilidad real de aprovechamiento térmico, ya que los productos obtenidos pueden ser utilizados en calderas y equipos industriales para el aprovechamiento energético.

El proceso de peletización ocasiona que el contenido de humedad de los residuos originales sea reducido, debido a que durante el procedimiento se da un aumento de la temperatura como consecuencia del rozamiento entre los rodillos y la matriz, con el respectivo calentamiento del producto y la evaporación de una porción de la humedad contenida [8].

Para un óptimo proceso de peletización es importante el control de variables tales como la humedad, temperatura, presión y otros.

Con la peletización es posible el aumento de las densidades aparentes de los residuos originales.

Las combinaciones de residuos se dan con distintas finalidades, desde la eliminación integral de todos los residuos hasta la obtención de un nuevo producto mejorado, pasando por diversas variantes de los residuos con más oportunidades de aprovechamiento comercial y energético.

\section{AGRADECIMIENTO}

A nuestros padres por darnos el apoyo a lo largo de estos años y a los amigos que hemos hecho en la universidad, en especial a nuestra amiga y compañera de estudio Ketzanys Samaniego, quien fue de gran apoyo en nuestro trabajo. A los profesores que nos dedicaron parte de su tiempo para transmitir sus conocimientos, pero especialmente al profesor Orlando Aguilar por brindarnos todo su conocimiento, paciencia y dedicación al confiarnos y participar con nosotros en este trabajo.

NOTA: Este artículo se terminó de editar y someter a publicación como homenaje póstumo a uno de sus autores, Gregory (Q.E.P.D.). Gregory espero que en el cielo tengas oportunidad de apreciar el fruto de tu trabajo y dedicación. Te recordamos en nuestro grupo.

\section{REFERENCIAS}

[1] K. Samaniego y A. Velásquez, Propuesta de Manejo Sostenible de los Residuos Sólidos generados en la Universidad Tecnológica de Panamá, Panamá, 2017.

[2] Instituto para la Diversificación y Ahorro de la Energía, «Energía de la Biomasa,» 2007.

[3] Stelte, J. Holm, A. Sanadi, S. Barsberg, J. Ahrenfeldt y U. Henriksen, «A study of bonding and failure mechanisms in fuel pellets from different biomass resources. Biomass and Bioenergy, $n^{\circ} 35$, pp. 910-918, 2011.

[4] S. García, Centrales Termoeléctricas de Biomasa, Renovetec. Madrid, 2012.

[5] S. Van Loo, J. Koppejan, Handbook of Biomass Combustion and Cofiring, Twente University Press, 2003.

[6] M. Bernanrdo, A. Santos, P. Cantinho, Cork industry wastewater partition by ultra/nanofiltration: a biodegradation and valorization Study. Water Research 45, 2011

[7] C. Sheng, J. Azevedo, Estimating the higher heating value of biomass fuels from basic analysis data, Biomass Bioenergy 28 , 2005.

[8] F. Sepúlveda, «Aprovechamiento Selectivo para la Valorización Integral de Residuos de la Industria del Corcho,» pp. 255-264, 2014. 